\title{
Parameter estimation for a phenomenological model of the cardiac action potential
}

\author{
F. Liu ${ }^{1} \quad$ J. Walmsley ${ }^{2} \quad$ K. Burrage ${ }^{3}$
}

(Received 28 December 2010; revised 14 July 2011)

\begin{abstract}
The action potential of a cardiac cell is made up of a complex balance of ionic currents which flow across the cell membrane in response to electrical excitation of the cell. Biophysically detailed mathematical models of the action potential have grown larger in terms of the variables and parameters required to model new findings in subcellular ionic mechanisms. The fitting of parameters to such models has seen a large degree of parameter and module re-use from earlier models. An alternative method for modelling electrically excitable cardiac tissue is a phenomenological model, which reconstructs tissue level action potential wave behaviour without subcellular details. A new parameter estimation technique to fit the morphology of the action potential in a four variable phenomenological model is presented. An approximation of a nonlinear ordinary differential equation model is established that corresponds to the given phenomenological model of the cardiac action potential. The parameter estimation problem is converted into a minimisation problem for the unknown parameters.
\end{abstract}

http://anziamj . austms.org.au/ojs/index.php/ANZIAMJ/article/view/3812 gives this article, (c) Austral. Mathematical Soc. 2011. Published July 28, 2011. IssN 1446-8735. (Print two pages per sheet of paper.) Copies of this article must not be made otherwise available on the internet; instead link directly to this URL for this article. 
A modified hybrid Nelder-Mead simplex search and particle swarm optimisation then solves the minimisation problem for the unknown parameters. The successful fitting of data generated from a well known biophysically detailed model is demonstrated. A successful fit to an experimental action potential recording that contains both noise and experimental artefacts is also produced. The parameter estimation method's ability to fit a complex morphology to a model with substantially more parameters than previously used is established.

\section{Contents}

1 Introduction

C483

2 Model structure

C486

3 Parameter estimation technique

C489

4 Results

C493

4.1 Example: Fitting Luo-Rudy $500 \mathrm{~ms}$ data and Luo-Rudy $1000 \mathrm{~ms}$ data

4.2 Example: Fitting to experimental data . . . . . . . . .

C493

5 Conclusions

C496

References

C497

\section{Introduction}

The action potential (AP) of a cardiac cell is caused by the flow of ions across the cell membrane through proteins known as ion channels. The opening and closing of many of these ion channels depends on the potential difference across the membrane, referred to as the membrane potential. The cell remains at a 
steady state or resting potential until the membrane potential is perturbed by either an injection of current into the cell through an electrode or a flow of charged ions from a neighbouring cell through gap junctions, a type of ion channel. This slight change in membrane potential causes the sodium channels to open which depolarizes the cell as positively charged sodium ions flow into the cell, inducing a sharp rise in the membrane potential known as the upstroke. Following the upstroke, an outward flux of potassium from the cell is opposed by a flow of calcium into the cell, producing a low gradient or plateau phase. Repolarisation of the cell and a return to the steady state potential results from the deactivation of the calcium current and the continuation of the potassium currents into the cell until the rest potential is reached. During the final stage of the AP, potassium and sodium ions are extruded from the cell through a series of pumps and ionic exchangers. If the cell is stimulated again before it has completed this process then the duration of the AP is shortened as there remains insufficient calcium outside the cell to counteract the outward flow of potassium. This property is known as AP duration restitution. The velocity and magnitude of the upstroke will also be reduced, slowing the conduction velocity of the AP in tissue, known as conduction velocity restitution. The phases of the AP are labelled in Figure $1[1]$.

The construction and parameterisation of a model of the electrical activity of a cardiac cell is a complex and difficult process. Mathematical models of the AP of a ventricular myocyte, the commonest electrically active cell in the heart, may feature as many as 67 variables [2] and include subcellular phenomena such as calcium handling and complex Markov formulations of ion channel gating kinetics. These biophysically detailed models have seen a large degree of parameter and module re-use from earlier models [3] which is inconsistent in factors such as species or temperature in the experiment performed. Often the data required to parameterise and validate a model for a particular current in the species in question cannot be found within the literature as it is either difficult or impossible to obtain, too time consuming to generate or is of insufficient interest to experimentalists. Given a valid 
parameter set, such models often need to be refitted to different regions of the same heart which can exhibit heterogeneity in electrophysiological properties, such as the conductances of various currents and the overall morphology of the AP.

An alternative method for modelling cardiac tissue is a phenomenological model [4, 5], which seeks to reconstruct the behaviour of the AP without resorting to the use of a biophysically detailed model. Properties such as AP morphology, dynamic restitution and conduction velocity restitution may all be fitted using this approach given appropriate experimental data from microelectrode or optical mapping experiments. The resulting model may then be used to investigate tissue level phenomena such as spiral waves during ventricular fibrillation.

A new parameter estimation technique to fit the morphology of the AP in the four variable, Bueno-Orovio phenomenological model [5] is proposed. The model is described in Section 2. The parameter space of the model, whilst significantly smaller than those in biophysically detailed models, is of large dimension (29 parameters). Many of these parameters have effects that only manifest themselves during certain temporal regions of the AP. In published parameter sets $[5,6]$ large ranges are seen in the values of parameters between different fits to the data. Furthermore, some parameters have a strong role in defining the AP morphology, whereas others control other properties such as dynamic restitution and thus have little effect on morphology. The parameter surface of the model therefore has regions of low and high sensitivity which vary with dimension and size of the parameter.

The parameter estimation of the model is converted into a minimisation problem for the unknown parameters. A modified hybrid Nelder-Mead simplex search and particle swarm optimisation is then used to minimise the objective function. This is discussed further in Section 3. Finally, both data generated from the Luo-Rudy model [7], paced at both $500 \mathrm{~ms}$ and $1000 \mathrm{~ms}$ cycle lengths, and an experimentally recorded AP from an isolated guinea pig ventricular myocyte [6] are used as target data for the minimisation process 
and to assess the success of the procedure by reference to a least squares objective function.

\section{$2 \quad$ Model structure}

The work presented in this section is based on the phenomenological model [5] of the ventricular AP. This phenomenological model is based upon the Fenton-Karma model [4]. This version of the model was chosen because the additional fourth variable provides the flexibility required to fit a wide variety of experimentally observed AP morphologies, whereas earlier versions were unable to reproduce AP morphology satisfactorily. As AP morphology is the focus of this paper this model property is the overriding concern.

The model consists of a total of four variables. The dimensionless voltage variable $u$ is rescaled to dimensions of millivolts $(\mathrm{mV})$ using the formula $V_{m V}=V u+R$. As $u$ generally takes the value zero at rest, $R$ is in effect the rest potential of the data, that is to say the transmembrane potential at which the cell sits at a steady state when exposed to no external stimulus at physiological extracellular ionic concentrations. The other three variables $v$, $w$ and $s$ act as gating variables, and control the activation and deactivation of the currents. The model equations are

$$
\begin{aligned}
& \frac{\partial u}{\partial t}=\nabla(D \nabla u)-\left(J_{\mathrm{fi}}+\mathrm{J}_{\mathrm{so}}+\mathrm{J}_{\mathrm{si}}+\mathrm{J}_{\mathrm{stim}}\right), \\
& \frac{\partial v}{\partial t}=\left[1-\mathrm{H}\left(\mathrm{u}-\theta_{v}\right)\right]\left(v_{\infty}-v\right) / \tau_{v}^{-}-\mathrm{H}\left(\mathrm{u}-\theta_{v}\right) v / \tau_{v}^{+}, \\
& \frac{\partial w}{\partial t}=\left[1-\mathrm{H}\left(\mathrm{u}-\theta_{w}\right)\right]\left(w_{\infty}-v\right) / \tau_{w}^{-}-\mathrm{H}\left(\mathrm{u}-\theta_{w}\right) w / \tau_{w}^{+}, \\
& \frac{\partial s}{\partial t}=\left(\left\{1+\tanh \left[k_{s}\left(u-u_{s}\right)\right]\right\} / 2-s\right) / \tau_{s},
\end{aligned}
$$

where $\mathrm{H}$ is the Heaviside function. The currents $\mathrm{J}_{\mathrm{fi}}, \mathrm{J}_{\mathrm{so}}$ and $\mathrm{J}_{\mathrm{si}}$ are

$$
\mathrm{J}_{\mathrm{fi}}=-v \mathrm{H}\left(u-\theta_{v}\right)\left(u-\theta_{v}\right)\left(u_{u}-u\right) / \tau_{f i},
$$




$$
\begin{aligned}
\mathrm{J}_{\mathrm{so}} & =\left(\mathrm{u}-\mathrm{u}_{\mathrm{o}}\right)\left[1-\mathrm{H}\left(\mathrm{u}-\theta_{w}\right)\right] / \tau_{\mathrm{o}}+\mathrm{H}\left(\mathrm{u}-\theta_{w}\right) / \tau_{\mathrm{so}}, \\
\mathrm{J}_{\mathrm{si}} & =-\mathrm{H}\left(\mathrm{u}-\theta_{w}\right) w \mathbf{s} / \tau_{\mathrm{si}},
\end{aligned}
$$

and $\mathrm{J}_{\text {stim }}$ is the applied stimulus current. Furthermore, some of the time constants themselves depend on the membrane potential as follows

$$
\begin{aligned}
& \tau_{v}^{-}=\left[1-\mathrm{H}\left(\mathrm{u}-\theta_{v}^{-}\right)\right] \tau_{v 1}^{-}+\mathrm{H}\left(\mathrm{u}-\theta_{v}^{-}\right) \tau_{v 2}^{-}, \\
& \tau_{w}^{-}=\tau_{w 1}^{-}+\left(\tau_{w 2}^{-}-\tau_{w 1}^{-}\right)\left\{1+\tanh \left[k_{w}^{-}\left(u-u_{w}^{-}\right)\right]\right\} / 2 \text {, } \\
& \tau_{\mathrm{so}}=\tau_{\mathrm{so} 1}+\left(\tau_{\mathrm{so} 2}-\tau_{\mathrm{so} 1}\right)\left\{1+\tanh \left[\mathrm{k}_{\mathrm{so}}\left(u-u_{\mathrm{so}}\right)\right]\right\} / 2, \\
& \tau_{s}=\left[1-\mathrm{H}\left(u-\theta_{w}\right)\right] \tau_{s 1}+\mathrm{H}\left(u-\theta_{w}\right) \tau_{s 2} \text {, } \\
& \tau_{\mathrm{o}}=\left[1-\mathrm{H}\left(\mathrm{u}-\theta_{\mathrm{o}}\right)\right] \tau_{\mathrm{o} 1}+\mathrm{H}\left(\mathrm{u}-\theta_{\mathrm{o}}\right) \tau_{\mathrm{o} 2} \text {. }
\end{aligned}
$$

The steady state values are

$$
\begin{aligned}
v_{\infty} & = \begin{cases}0, & u<\theta_{v}^{-}, \\
1, & u \geqslant \theta_{v}^{-},\end{cases} \\
w_{\infty} & =\left(1-\mathrm{H}\left(u-\theta_{0}\right)\right)\left(1-u / \tau_{w \infty}\right)+\mathrm{H}\left(u-\theta_{0}\right) w_{\infty}^{*} .
\end{aligned}
$$

The model employs a diffusion term in Equation (1) and so is similar to the monodomain equations used to simulate cardiac electrophysiology for biophysically detailed models [8]. However, in the course of simulation, diffusion is taken to be constant at $1.171 \mathrm{~cm}^{2} / \mathrm{s}$, based on experimental measurements of human ventricular cell size and conductances. Thus, the relation in (1) becomes the Laplacian as the constant diffusion coefficient may be moved outside of the gradient operator. It is noted that this model can equally well take into account factors such as fibre orientation by setting the diffusion parameter $\mathrm{D}$ to be a conductivity tensor, at which point this property will no longer hold [4]. As the parameter estimation method is concerned only with AP morphology, D $=0$ is used in this study.

The currents represent an approximation to groups of currents observed in the cell rather than having a biophysical meaning. The behaviour of the model is shown in Figure 1 using the human epicardial cell parameter set from 

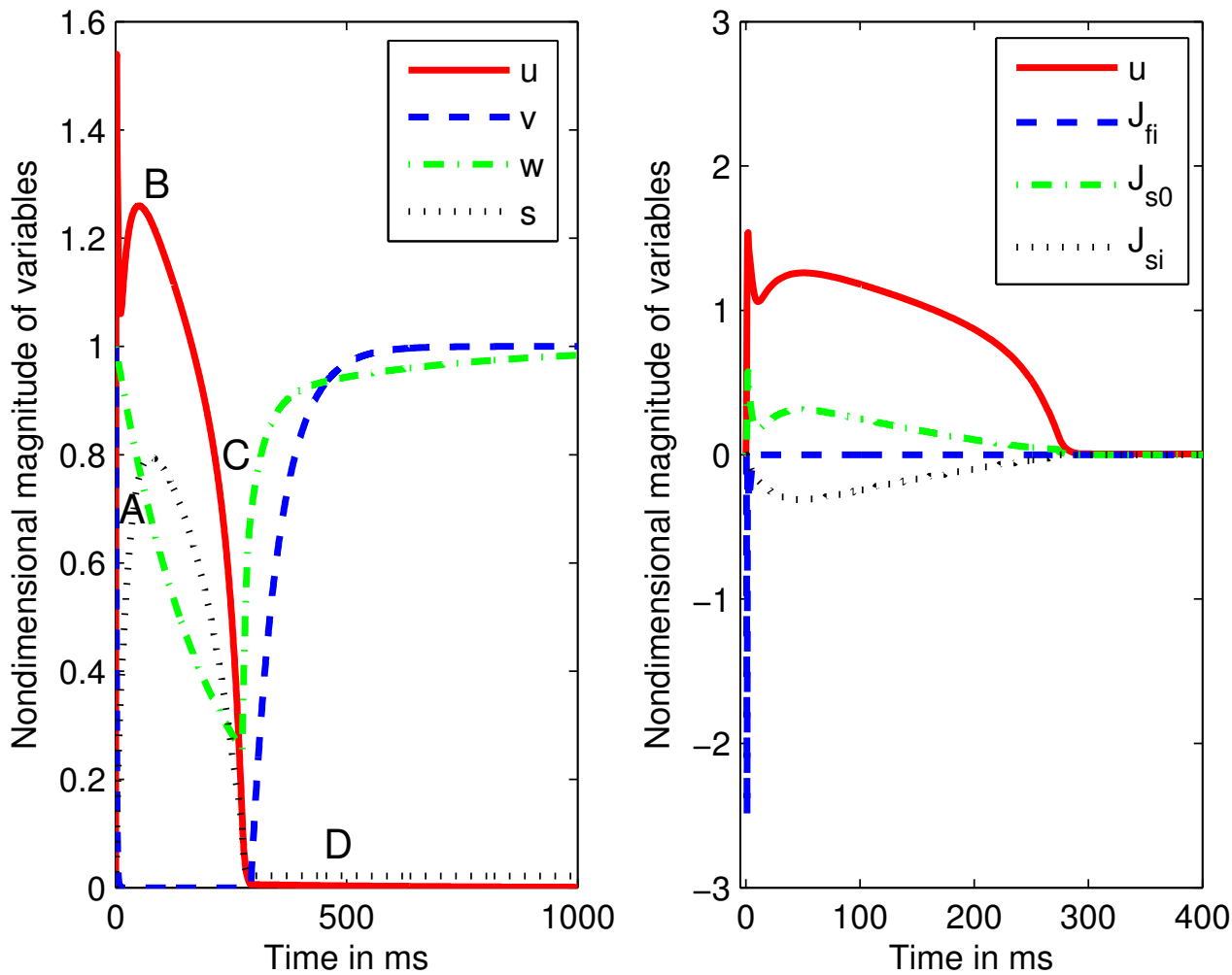

Figure 1: Simulated data using the epicardial data set provided after five stimuli to reach steady state at a pacing frequency of $1000 \mathrm{~ms}$. The variables of the model $u, v, w$ and $s$ and the currents $\mathrm{J}_{\mathrm{fi}}, \mathrm{J}_{\text {so }}$ and $\mathrm{J}_{\mathrm{si}}$ over the same AP are shown. In the first figure, the four basic phases of the AP are shown against the variable $u$. The point $\mathrm{A}$ marks the upstroke, $\mathrm{B}$ the plateau phase, $\mathrm{C}$ repolarization, and $\mathrm{D}$ the resting phase.

Bueno-Orovio et al. [5] in response to a stimulus current of non-dimensional magnitude -0.5 and of duration $2 \mathrm{~ms}$. The behaviour of the potential $u$, the gating variables $v, w$ and $s$ and the currents $\mathrm{J}_{\mathrm{fi}}, \mathrm{J}_{\mathrm{so}}$ and $\mathrm{J}_{\mathrm{si}}$ are shown. The same stimulus magnitude and duration are used for the parameter fitting process. 
This model reproduces physiological properties such as maximum velocity of upstroke, threshold of excitation, AP morphology, dynamic restitution curves and conduction velocity restitution curves.

\section{Parameter estimation technique}

Nonlinear programming methods have been applied to solve the global optimization of continuous-variable functions [9]. Searching a continuous-variable function under a given search domain must locate the global optimum without being trapped in local solutions. Optimization techniques can be classified into two broad categories: traditional direct search techniques (such as simplex search methods) and evolutionary techniques (such as the particle swarm optimization). The Nelder-Mead method [10] is a simple direct search technique that has been widely used in various unconstrained optimization scenarios. One of the reasons for its popularity is that the method is easy to implement and does not need the derivatives of the function under exploration. However, one has to be very careful when using the Nelder-Mead method since it can be sensitive to the choice of initial points and is not guaranteed to attain the global optimum. Eberhart and Kennedy [11] proposed a new heuristic algorithm called particle swarm optimization (PSO). The theory of PSO lets each particle fly through a multidimensional search space while the particles' velocity and position are constantly updated based on the best previous performance of the particle and of the particles' neighbours, as well as the best performance of particles in the entire population. PSO has been successful in optimizing various continuous nonlinear functions, including nonlinear unknown parameters and unknown domains of parameters.

A new parameter estimation technique is proposed, that is, a modified hybrid Nelder-Mead simplex search and a particle swarm optimization method to fit the morphology of the AP in the four variable Bueno-Orovio phenomenological model. 
The governing deterministic dynamical system can be written as a system of differential equations with $m$ unknown parameters,

$$
\begin{aligned}
\frac{d y(t)}{d t} & =f\left(t, y, \lambda_{1}, \lambda_{2}, \ldots, \lambda_{m}\right), \quad 0 \leqslant t \leqslant T, \\
y(0) & =y^{0},
\end{aligned}
$$

where $y=\left(y_{1}, y_{2}, \ldots, y_{n}\right)^{\top}$ and $f=\left(f_{1}, f_{2}, \ldots, f_{n}\right)^{\top}$ are $n$-dimensional vector functions, $f_{i}(i=1,2, \ldots, n)$ may be nonlinear with respect to the unknown parameters $\lambda_{i}(i=1, \ldots, m), m$ is the number of parameters, $n$ is the number of species.

The differential/algebraic system solver was used to solve the system of differential equations. The differential/algebraic system is based on the backward difference formulas, and approximates the derivatives using the $k$ th order backward difference formulas, where $k$ ranges from one to five. At every step it chooses the order $k$ and stepsize based on the behaviour of the solution.

Let $\left(\lambda_{1}, \lambda_{2}, \ldots, \lambda_{m}\right) \in R$, where $R$ is a bounded domain of the form

$$
\mathrm{R}=\left[\lambda_{1}^{(\min )}, \lambda_{1}^{(\max )}\right] \times\left[\lambda_{2}^{(\min )}, \lambda_{2}^{(\max )}\right] \times \cdots \times\left[\lambda_{\mathrm{m}}^{(\min )}, \lambda_{\mathrm{m}}^{(\max )}\right] .
$$

Let $y_{i}\left(t_{k}\right)$ be given target solutions of (15) and (16), while the $y_{i, k}$ are numerical solutions of (15) and (16) using differential/algebraic system solver for given $\lambda=\left(\lambda_{1}, \lambda_{2}, \ldots, \lambda_{m}\right) \in R$. The error of the given parameter vector $\lambda^{*}=\left(\lambda_{1}^{*}, \lambda_{2}^{*}, \ldots, \lambda_{\mathrm{m}}^{*}\right) \in \mathrm{R}$ of $(15)$ is

$$
\underline{g}\left(\lambda^{*}\right)=\min _{\lambda \in R} \underline{g}(\lambda)=\min _{\lambda \in R}\left\{\frac{1}{n} \sum_{i=0}^{n}\left[\sqrt{\frac{\sum_{k=0}^{N}\left(y_{i}\left(t_{k}\right)-y_{i, k}\right)^{2}}{N+1}}\right]\right\}
$$

where $T=N \tau$ and $\tau$ is a time step.

A modified hybrid Nelder-Mead simplex search and a particle swarm optimization method that tries to find a potential global minimum $g\left(\lambda^{*}\right)$ of a multimodal, continuous-variable function in (15) is now described. 
The novel MH-NMSS-PSO is based on the Nelder-Mead simplex search method (NMSS) [10] and the particle swarm optimization (PSO) algorithm [11] for the optimization of multimodal functions. The NMSS focuses on "exploitation"; the PSO focuses on "exploration". The first major difference between NMSS and PSO is the choice of initial points. In the NMSS, the initial points are pre-determined, but they are a set of random points in PSO. The second difference is with the directions and conditions of the preceding steps. The PSO proceeds by moving towards those points that have better (objective) function values, whereas the NMSS evolves by moving away from a point that has the worst performance.

Taking the better characteristics of each method, a modified hybrid NelderMead simplex search and particle swarm optimization method is proposed.

An initial population, $3 m+1$ particles, is constructed in two parts. Firstly, the standard starting point is used in the NMSs to form an initial simplex of $m+1$ particles, and an additional $2 m$ particles are randomly generated in the PSO part. The population of $2 \mathrm{~m}$ particles in the PSO part may be a worthy investment as they may bring about an early convergence to the vicinity of the global optimum.

A total of $3 m+1$ particles are sorted by their objective function value $\underline{g}\left(\lambda^{*}\right)$ in (18), and the best $m$ particles are saved for subsequent use in the simplex search part of the hybrid method. Joined by the $m$ best particles and the $(m+1)$ th particle, the last $2 m$ particles are adjusted by the PSO method (that is, selection and velocity update). The procedure for adjusting the last $2 \mathrm{~m}$ particles involves selection of the global best particle, selection of the neighbourhood best particles, and finally velocity updates.

The modified hybrid Nelder-Mead simplex search and particle Swarm optimization (MH-NMSS-PSO) algorithm is summarised in Algorithm 1. 


\section{Algorithm 1: $\mathrm{MH}-\mathrm{NMSS}-\mathrm{PSO}$}

Input: Intervals

$R=\left[\lambda_{1}^{(\min )}, \lambda_{1}^{(\max )}\right] \times\left[\lambda_{2}^{(\min )}, \lambda_{2}^{(\max )}\right] \times \cdots \times\left[\lambda_{\mathrm{m}}^{(\min )}, \lambda_{\mathrm{m}}^{(\max )}\right]$ to

search for solution within, initial velocities, given target

solutions $y_{i}\left(t_{k}\right),(i=1, \ldots, m ; k=1, \ldots, n)$, the error

parameter $\epsilon$ and the number of iterations $\mathrm{N}_{\text {iter }}$.

Output: The best parameter estimation values $\lambda^{*}=\left(\lambda_{1}^{*}, \lambda_{2}^{*}, \ldots, \lambda_{\mathrm{m}}^{*}\right)$.

1 Generate a population of size $3 m+1$;

2 for $I T=1: \mathrm{N}_{\text {iter }}$ do

$3 \quad$ (i) Evaluation and Ranking: evaluate the objective function value $\underline{g}(\lambda)$ in (18) of each particle;

4 (ii) Nelder-Mead simplex search method: apply a NMSS operator to the best $m+1$ particles and replace the $(m+1)$ th particle with the update;

5 (iii) Particle Swarm Optimisation: apply the PSO operator for updating $2 \mathrm{~m}$ particles with the worst objective function value;

6 if the stopping criterion $S_{c}<\epsilon$ then

7 break;

8 end

9 end

The stopping criterion is then

$$
\mathrm{S}_{\mathrm{C}}=\left[\sum_{i=1}^{\mathrm{m}+1} \frac{\left(\overline{\mathrm{g}}-\sqrt{\mathrm{g}_{\mathrm{i}}}\right)^{2}}{\mathrm{~m}+1}\right]^{1 / 2}<\epsilon
$$

where

$$
g_{i}^{*}=\underline{g}\left(P_{i}\right)=\sqrt{g_{i}}=\sqrt{g_{i}\left(\lambda_{1, i}, \lambda_{2, i}, \ldots, \lambda_{m, i}\right)}, \quad \bar{g}=\sum_{i=1}^{m+1} \frac{g_{i}^{*}}{m+1},
$$

and $\epsilon$ is a small error parameter. 
All numerical methods and MH-NMSS-PSO have been implemented in Fortran 77 on a PC.

\section{Results}

The MH-NMSS-PSO algorithm and numerical technique are employed to estimate the parameters for the phenomenological model of the AP described in Section 2. The ranges used for our parameter estimation are based on [5] and behaviour of the potential $u$.

\subsection{Example: Fitting Luo-Rudy $500 \mathrm{~ms}$ data and Luo-Rudy $1000 \mathrm{~ms}$ data}

Using the MH-NMSS-PSO algorithm and numerical technique, parameter estimates are obtained to fit the phenomenological model to data generated by the Luo-Rudy model [7] paced at both $500 \mathrm{~ms}$ and $1000 \mathrm{~ms}$ cycle lengths. The dimensionless voltage variable $u$ is rescaled to dimensions of Millivolts using the equation $\mathrm{V}_{\mathrm{m} v}=\mathrm{Vu}+\mathrm{R}$ to produce the final fits to the data that are shown in Figure 2. The estimated parameter values are listed in Table 1. Here PA denotes parameter and GP denotes Guinea Pig.

\subsection{Example: Fitting to experimental data}

Using the MH-NMSS-PSO algorithm and numerical technique, parameter values are obtained to fit the phenomenological model to an experimentally recorded AP from an isolated guinea pig ventricular myocyte paced at a cycle length of $1000 \mathrm{~ms}$. The experimental data used in this study has previously been detailed by Walmsley et al. [6]. The experiment was conducted in accordance 

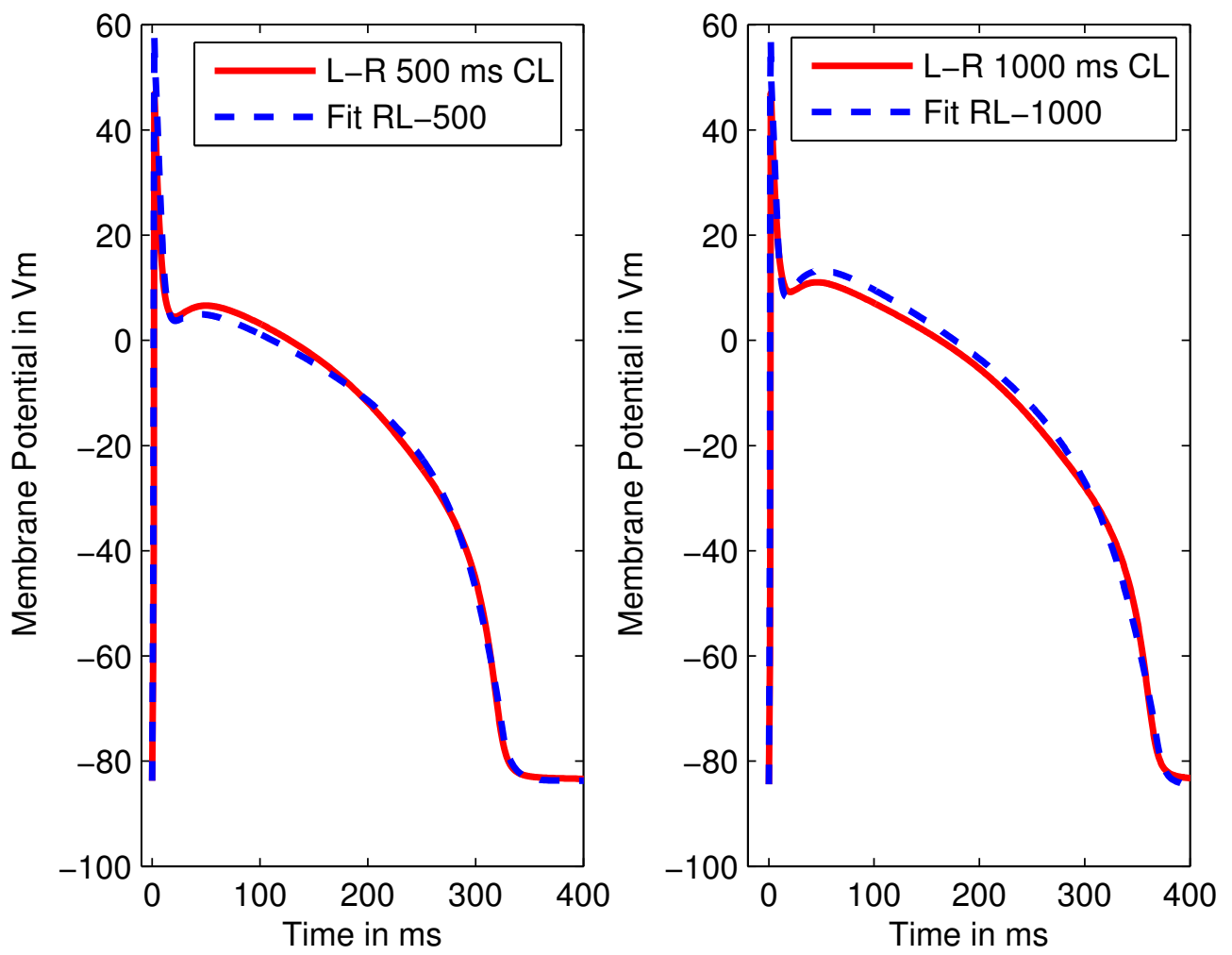

FiguRE 2: A comparison of the deterministic model obtained by refitting the parameters of the original model [5] to a sample AP generated by the Luo-Rudy model at cycle lengths of $500 \mathrm{~ms}$ and $1000 \mathrm{~ms}$. The first figure has a $500 \mathrm{~ms}(2 \mathrm{~Hz})$ cycle length and the second figure has a $1000 \mathrm{~ms}(1 \mathrm{~Hz})$ cycle length. The horizontal axis has been truncated at $400 \mathrm{~ms}$ to better display the fit. 

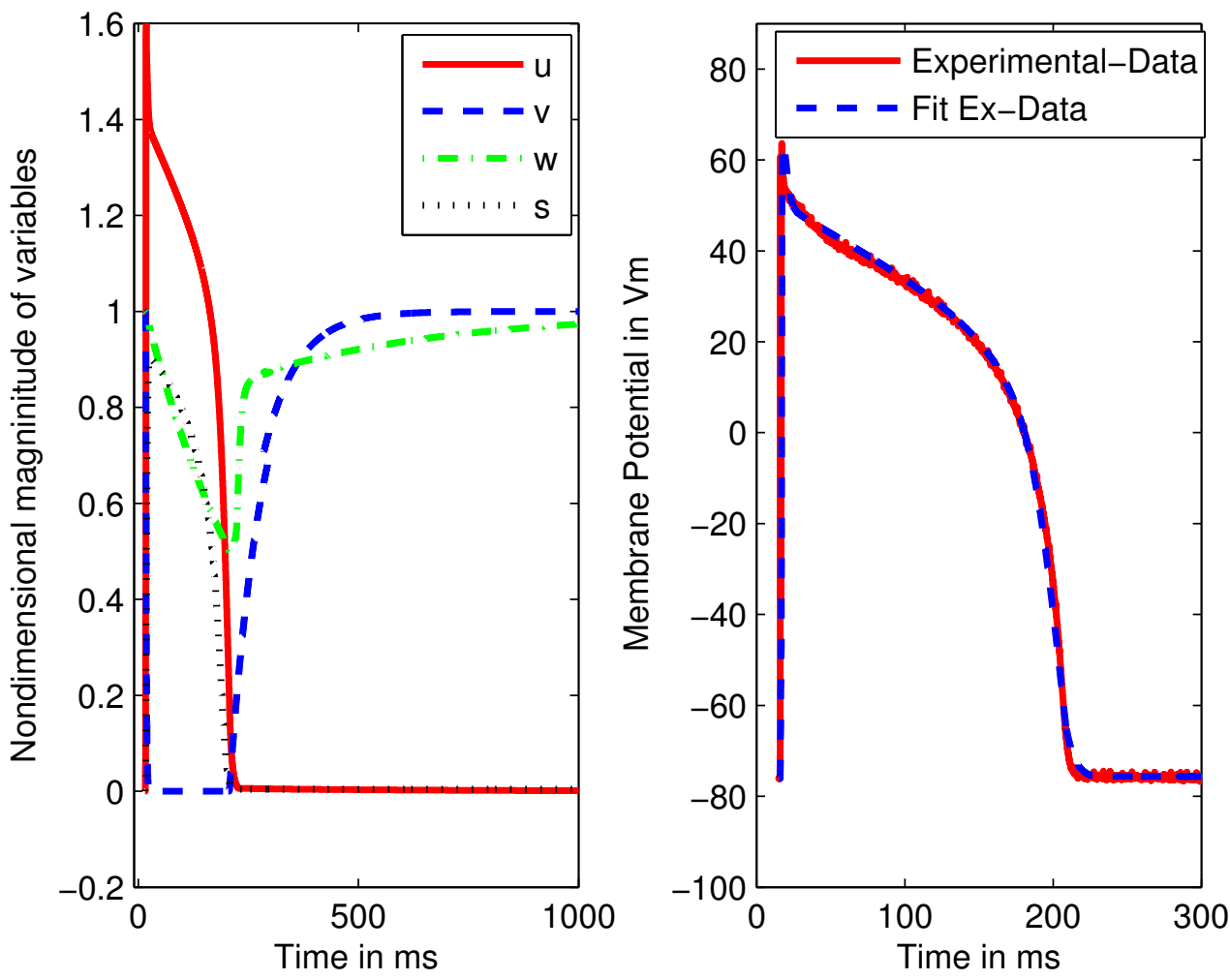

Figure 3: A comparison of the deterministic model obtained by refitting the parameters of the original model to a sample AP drawn from the experimental data for a guinea pig isolated ventricular myocyte. The horizontal axis has been truncated at $300 \mathrm{~ms}$ to better display the fit in the second figure. 
TABLE 1: Fitted parameter values

\begin{tabular}{|lrrr|lrrr|}
\hline PA & LR 500 & LR 1000 & GP & PA & LR 500 & LR 1000 & GP \\
\hline$\theta_{v}$ & 0.4509 & 0.3647 & 0.3200 & $\theta_{w}$ & 0.0917 & 0.1265 & 0.1300 \\
$\theta_{v}^{-}$ & 0.0000 & 0.0060 & 0.1900 & $\theta_{\mathrm{o}}$ & 0.0000 & 0.0003 & 0.0060 \\
$\mathrm{u}_{\mathrm{s}}$ & 0.9185 & 0.9324 & 0.9800 & $\mathrm{u}_{w}^{-}$ & 0.0000 & 0.0251 & 0.0175 \\
$\mathrm{u}_{\mathrm{so}}$ & 0.7785 & 0.6324 & 0.7072 & $\mathrm{u}_{\mathrm{u}}$ & 1.9180 & 1.7530 & 1.6200 \\
$\mathrm{u}_{\mathrm{o}}$ & 0.0000 & 0.0000 & 0.0000 & $\tau_{w}^{\infty}$ & 0.09323 & 0.0865 & 0.0410 \\
$\tau_{\mathrm{o} 1}$ & 571.000 & 521.2769 & 456.3476 & $\tau_{\mathrm{o} 2}$ & 8.8481 & 6.6474 & 6.1000 \\
$\tau_{\mathrm{s} 1}$ & 4.1762 & 2.7342 & 3.0600 & $\tau_{\mathrm{s} 2}$ & 12.5582 & 13.2947 & 2.1900 \\
$\tau_{\mathrm{so}}$ & 44.1989 & 56.4735 & 30.9000 & $\tau_{\mathrm{s} 2}$ & 1.3781 & 1.2295 & 1.2874 \\
$\tau_{w 1}^{-}$ & 58.2595 & 53.2368 & 6.1828 & $\tau_{w 2}^{-}$ & 18.2595 & 125.8942 & 140.000 \\
$\tau_{v 1}^{-}$ & 85.8577 & 71.7017 & 76.0000 & $\tau_{v 2}^{-}$ & 973.0652 & 13.4584 & 10.300 \\
$\tau_{s i}$ & 5.0682 & 3.8715 & 2.7844 & $\tau_{v}^{+}$ & 2.5500 & 2.1623 & 1.3500 \\
$\tau_{w}^{+}$ & 371.0585 & 348.6196 & 276.000 & $\tau_{\mathrm{fi}}$ & 0.2369 & 0.1809 & 0.1100 \\
$w_{\infty}^{*}$ & 0.8523 & 0.8266 & 0.8430 & $\mathrm{k}_{\mathrm{so}}$ & 2.2857 & 2.0647 & 2.0100 \\
$\mathrm{k}_{w}^{-}$ & 61.9641 & 152.3928 & 206.9896 & $\mathrm{k}_{\mathrm{s}}$ & 2.0874 & 2.0952 & 2.8100 \\
$\mathrm{~V}$ & 77.5000 & 81.6499 & 90.5000 & $\mathrm{R}$ & -83.720 & -84.400 & -76.100 \\
\hline
\end{tabular}

with the UK Home Office guidance on the Operation of Animals (Scientific Procedures) Act of 1986.

The estimated parameter values are shown in Table 1. The dimensionless voltage variable $u$ is rescaled to dimensions of millivolts using the equation $\mathrm{V}_{\mathrm{m} V}=\mathrm{Vu}+\mathrm{R}$ to produce the final fit to the experimental data, shown together with nondimensional magnitude of current variables in Figure 3.

The simulations produced give an excellent agreement with both the generated AP morphologies from the Luo-Rudy model and the experimental data.

\section{Conclusions}

The modified hybrid Nelder-Mead simplex search and particle swarm optimization is a valid method for the fitting of AP morphology in a cardiac electrophysiology tissue model. The closeness of fit achieved by the model 
to data both artificially generated from a biophysically detailed cardiac electrophyslogy model and experimental data taken from an isolated ventricular myocyte has been shown. AP duration restitution properties, as described in Section 1, are not recovered adequately but this was not an aim of this study. This is shown by the variation in parameters between the $500 \mathrm{~ms}$ and $1000 \mathrm{~ms}$ columns in Table 1. Ideally, one set of parameters would describe both sets of data. The different results obtained indicate that fitting the model to an AP morphology derived from one cycle length is insufficient to fully parameterise all aspects of the model using this method. Extra data at different cycle lengths is required to do this. The program runs to a successful completion in a few minutes for the initial parameter ranges. However, the efficacy of this approach is very much dependent on the choice of initial ranges for the parameters. These techniques can be applied to the parameter estimation of other kinds of differential algebraic equations [12].

Acknowledgements We thank Dr. Christian Bollensdorff and Maya Bahoshy of the Department of Physiology, Anatomy and Genetics, University of Oxford, UK, for the provision of experimental data for an isolated guinea pig ventricular myocyte. John Walmsley is funded by the Engineering and Physical Sciences Research Council through the Systems Biology DTC.

\section{References}

[1] E. Carmeliet and J. Vereecke, Cardiac Cellular Electrophysiology, Springer, 2002. C484

[2] V. Iyer, R. Mazhari and R. Winslow, A computational model of the human left-ventricular epicardial myocyte, Biophys. J., 87, 2004, 1507-1525, doi:10.1529/biophysj.104.043299 C484 
[3] S. Niederer, M. Fink, D. Noble and N. P. Smith, A meta-analysis of cardiac electrophysiology computational models, Exp. Physiol., 94, 2009, 486-495, doi:10.1113/expphysiol.2008.044610 C484

[4] F. Fenton, and A. Karma, Vortex dynamics in three-dimensional continuous myocardium with fiber rotation: Filament instability and fibrillation, Chaos, 8, 1998, 20-47, doi:10.1063/1.166311 C485, C486, C487

[5] A. Bueno-Orovio, E. M. Cherry and F. H. Fenton, Minimal model for human ventricular action potentials in tissue, J. Theor. Biol., 253, 2008, 544-560, doi:10.1016/j.jtbi.2008.03.029 C485, C486, C488, C493, C494

[6] J. Walmsley, G. Mirams, M. Bahoshy, C. Bollensdorff, B. Rodriguez and K. Burrage, Phenomenological modeling of cell-to-cell and beat-to-beat variability in isolated Guinea Pig ventricular myocytes, Conf. Proc. IEEE Eng. Med. Biol. Soc. 2010, 2010, 1457-60, doi:10.1109/IEMBS.2010.5626858 C485, C493

[7] C. Luo and Y. Rudy, A model of the ventricular cardiac action potential. Depolarization, repolarization, and their interaction, Circ. Res., 68, 1991, 1501-1526, doi:10.1161/01.RES.68.6.1501 C485, C493

[8] J. Keener and J. Sneyd, Mathematical Physiology, Springer, 1998. C487

[9] M. M. Macconi, B. Morini and M. Porcelli, A Gauss-Newton method for solving bound-constrained underdetermined nonlinear systems, Optimization Method and Software, 24(2), 2010, 219-235, doi:10.1080/10556780902753031 C489

[10] J. A. Nelder and R. A. Mead, A simplex method for function minimization, Computer Journal, 7, 1965, 308-313, doi:10.1093/comjnl/7.4.308 C489, C491

[11] R. C. Eberhart and J. Kennedy, A new optimizer using particle swarm theory, Proc. of the Sixth International Symposium on Micro Machine 
and Human Science, Nagoya, Japan, 1995, 39-43,

doi:10.1109/MHS.1995.494215 C489, C491

[12] F. Liu and K. Burrage, Novel techniques in parameter estimation for fractional dynamical models arising from biological systems, Computers and Mathematics with Applications, 2011, doi:10.1016/j.camwa.2011.03002 C497

\section{Author addresses}

1. F. Liu, Mathematical Sciences, Queensland University of Technology, Brisbane, Queensland 4001, Australia. mailto:f.liu@qut.edu.au

2. J. Walmsley, Department of Computer Science, University of Oxford, Wolfson Building, Parks Road, Oxford, OX1 3QD, UK.

3. K. Burrage, Department of Computer Science and OCISB, University of Oxford, OX1 3LB, UK; Queensland University of Technology, Brisbane, Australia. 